\title{
Design of Fuzzy Temperature Control System Based on LabVIEW
}

\author{
Niu Ruiyan ${ }^{\mathrm{a},{ }^{* 1}}$, Ren Junjie ${ }^{\mathrm{a}, * 2}$ \\ ${ }^{a}$ College of Automation, Beijing Union University, Beijing, China
}

\begin{abstract}
In this paper, an effective method of design fuzzy logic controller is introduced based on LabVIEW. The steps of setting membership function, fuzzy rules and fuzzy interference are introduced in details of design fuzzy controller. It is implemented in the oven temperature control system, which has practical value for engineering.
\end{abstract}

Index Terms: Virtual Instrument; Fuzzy Logic; Thermocouple

(C) 2011 Published by MECS Publisher. Selection and/or peer review under responsibility of the International Conference on E-Business System and Education Technology

\section{Introduction}

Virtual Instrument (LabVIEW) is that virtual panel is customized by the user, and measuring and controlling function is be completed by using computer. The basic idea of fuzzy control is to use a computer to achieve human experience, and these experiences are mostly expressed in the language of considerable ambiguity of the rules. Fuzzy controller has adaptive ability, robustness and stability because of its logic, so it is suitable for the system which hasn't precise mathematical model.

\section{System Components}

In the paper, fuzzy logic is applied to the temperature control system based on virtual instrument. Using the thermocouple to measure the temperature of the oven, we compare it with the given value. When the error which is generated by the difference between the measuring temperature and set temperature is greater than 6 ${ }^{0} \mathrm{C}$, the timer produces a larger duty-cycle pulse sequence. Otherwise the fuzzy controller produces duty cycle of pulse sequence according to the error and its change rate, and then by the solid-state relay controlling the power of the oven, therefore the temperature of the oven would be changed. Block diagram of the temperature control system is shown in Fig.1.

Corresponding author:

| E-mail address: ${ }^{* 1} \underline{\text { zdhtruiyan @ buu.edu.cn; }}{ }^{* 2}$ zdhtjunjie@ buu.edu.cn 


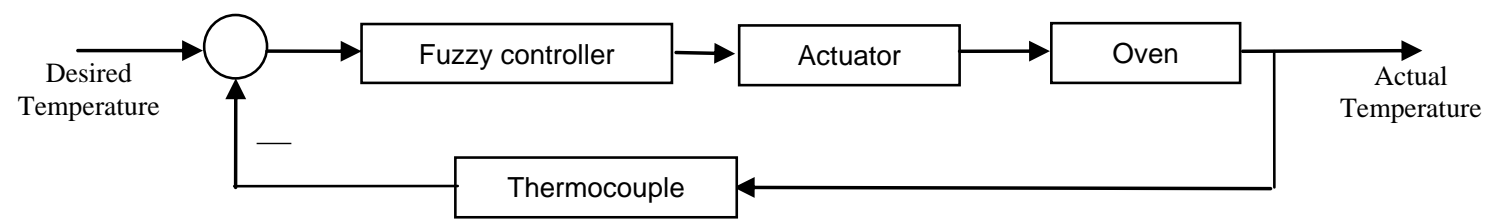

Fig. 1. Block diagram of the temperature control system

\section{Temperature Measurement}

\subsection{Data Acquisition}

According to the difference between positive and negative electrode materials, the thermocouple is divided into B, E, J, K, R, S, T, Y-type. We use a K-type thermocouple which is linear better, and it has been widely used in industrial measurement. We should take the cold side temperature of the thermocouple into account, that is the cold side compensation.

\subsection{Signal conditioning}

Because voltage signal generated by thermocouple is very low, it needs further amplification, filtering, and linear. In this paper, SCC-TC02 (thermocouple conditioning module) is connected with data acquisition card through the SC-2345 shielded box. SCC-TC02 module schematic diagram is shown in Fig.2.

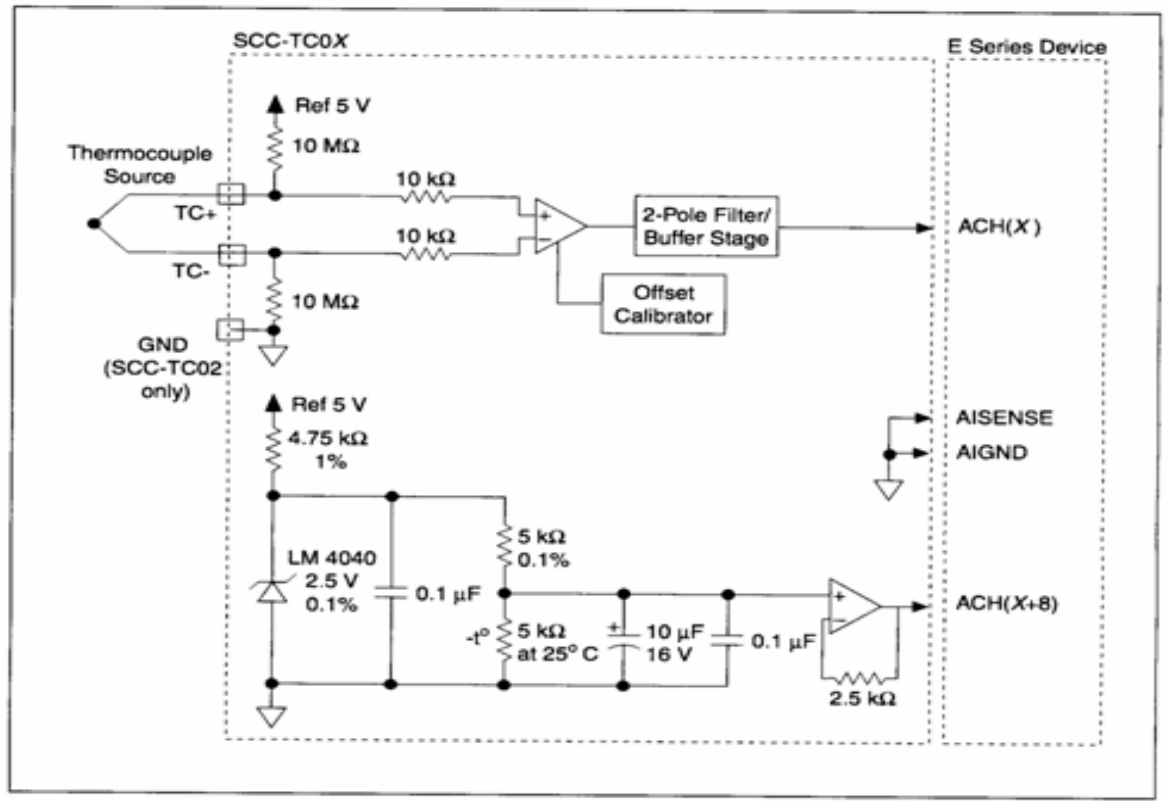

Fig. 2. SCC-TC02 Thermocouple conditioning module schematic diagram 
SCC-TC02 has three interfaces: TC+, TC-, and GND. TC+ is the positive of the thermocouple and TC- is the negative. Ground terminal is connected to AIGND of the E Series DAQ devices. The amplified thermocouple signal and cold junction sensor signal are measured by E Series DAQ device channel $\mathrm{X}$ and channe $1 \mathrm{X}+8$ respective1y, where $\mathrm{X}$ is 0 to 7 depending on where you plug the SCC-TC02.

Thermocouple conditioning module consists of two parts. One part of it is connected to the thermocouple, which has a amplifier with a gain of 100 and filters; the other part is the circuit which room temperature is measured by thermistor. We can use the formulas to convert the cold junction sensor voltage to cold junction temperature.

\section{Data Processing}

The data of thermocouple signal and cold junction sensor signal are measured by DAQ device channel X and channe $1 \mathrm{X}+8$ respective1y is in a array, then through Index of the array to separate them and dealt with them separately .

\subsection{Thermocouple signal Processing}

The thermocouple temperature and voltage relationship is nonlinear, so the temperature is divided into several sections. In each section, the temperature and voltage relationship is linear, you can use (1) to calculate the temperature corresponding to the voltage values based on K-type thermocouple table.

$$
T=\frac{T_{2}-T_{1}}{V_{2}-V_{1}} \times\left(V-V_{1}\right)+T
$$

Thermocouple signal processing subroutine uses a case structure[1-3], shown in Fig. 3:

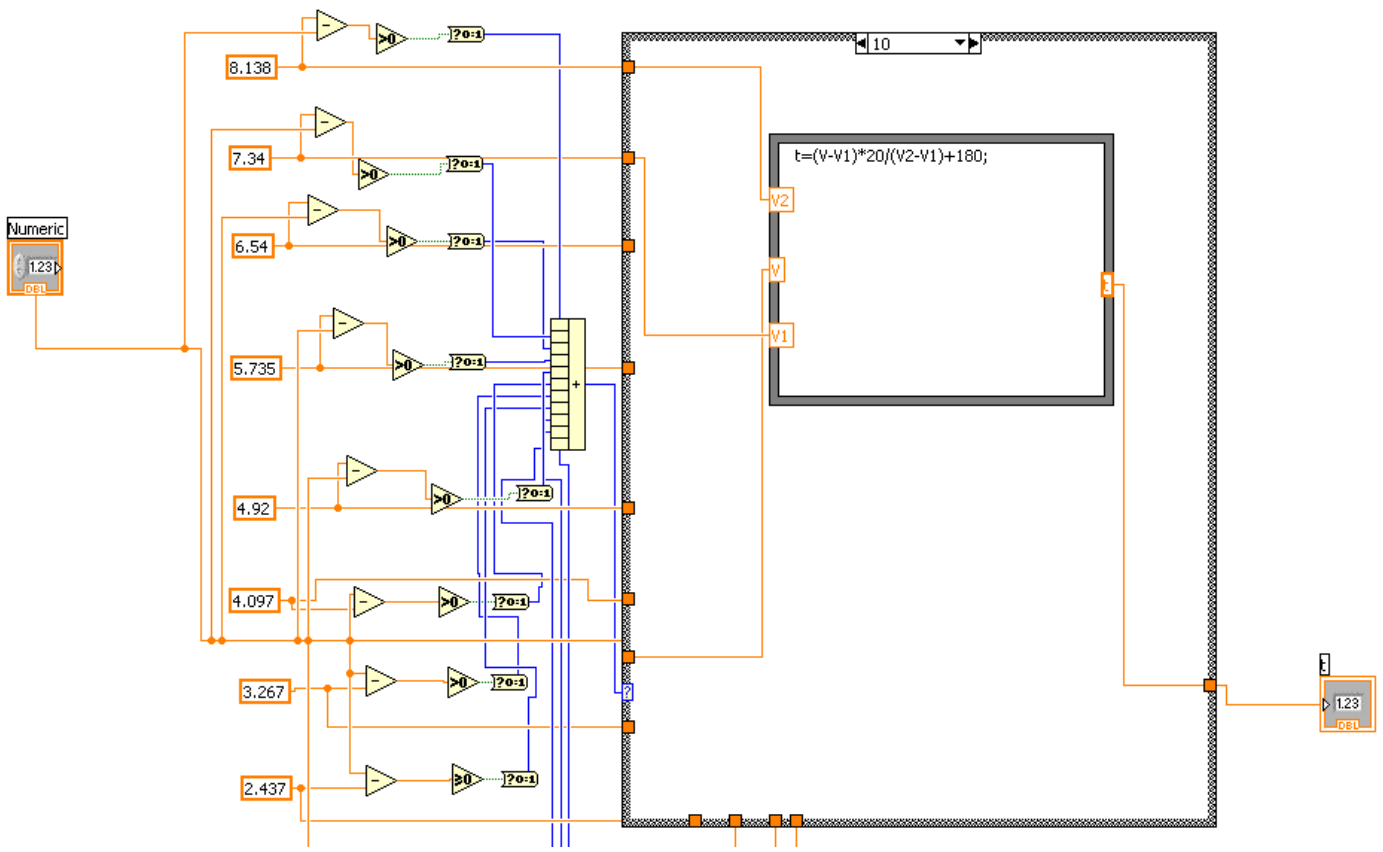

Fig. 3. Thermocouple signal processing subroutine 


\subsection{The cold junction sensor voltage processing}

You can use the following formulas to convert the cold junction sensor voltage to cold junction temperature:

$$
\mathrm{T}\left({ }^{0} \mathrm{C}\right)=\mathrm{T}_{\mathrm{K}}-273.15
$$

Where

$$
T_{K}=\frac{1}{\left[\mathrm{a}+\mathrm{b}\left(\ln \mathrm{R}_{\mathrm{T}}\right)+\mathrm{c}\left(\ln \mathrm{R}_{\mathrm{T}}\right)^{3}\right]}
$$

$$
\begin{aligned}
& a=1.295361 \times 10^{-3} \\
& b=2.343159 \times 10^{-4} \\
& c=1.018703 \times 10^{-7}
\end{aligned}
$$

$\mathrm{R}_{\mathrm{T}}$ is the resistance of the thermistor in ohms.

$$
R_{T}=5000\left(\frac{V_{\text {TEMPOUT }}}{2.5-V_{\text {TEMPOUT }}}\right)
$$

Where

$\mathrm{V}_{\text {TEMPOUT }}$ is the output voltage of the cold junction sensor.

$$
T\left({ }^{\circ} \mathrm{F}\right)=\frac{\left[T\left({ }^{\circ} \mathrm{C}\right)\right] 9}{5}+32
$$

Where

$\mathrm{T}\left({ }^{\circ} \mathrm{F}\right)$ and $T\left({ }^{\circ} \mathrm{C}\right)$ are the temperature readings in degrees Fahrenheit and Celsius, respectively.

\section{Fuzzy Controller Design}

Design of fuzzy control needs five steps:

\subsection{Determining input and output linguistic variables of the fuzzy controller}

Because the error and its change rate are the fuzzy controller input variables, and output variable is a fuzzy controller output, fuzzy controller is a dual-input single-output type.

\subsection{Fuzzy process}

Fuzzy linguistic variables which are E, EC and U describe the error, error change rate and fuzzy controller output respectively[4]. Because the oven can not be cooled in operation, if the oven temperature is greater than the given value, duty cycle of pulse sequence is zero, so the error is only positive. The domain of 6 degrees 
Celsius is divided into seven sections, namely: (Very-Small, Small, Medium-Small, Medium, Middle-Big, Big Very-Big), denoted by $\mathrm{E}=(\mathrm{VS}, \mathrm{S}, \mathrm{MS}, \mathrm{M}, \mathrm{MB}, \mathrm{B}, \mathrm{VB})$, the domain of $\mathrm{E}$ is $(0,+1,+2,+3,+4,+5,+6)$. By the same, the error change rate is divided into seven sections, namely $\mathrm{EC}=\{$ Negative-Big, Negative-Medium, Negative-Small, Zero, Positive-Small, Positive-Medium, Positive-Big $\}=\{$ NB, NM, NS, ZR, PS, PM, PB $\}$, the domain of EC is $(-3,-2,-1,0,+1,+2,+3)$. Output variable $U$ is also divided into seven sections, namely $\mathrm{U}=\{$ Very-Small, Small, Medium-Small, Medium, Medium-Big, Big Very-Big $\}=\{\mathrm{VS}, \mathrm{S}, \mathrm{MS}, \mathrm{M}, \mathrm{MB}, \mathrm{B}$, $\mathrm{VB}\}$, the domain of $\mathrm{U}$ is $(0,+1,+2,+3,+4,+5,+6)$. Scaling factors is $\mathrm{Ke}, \mathrm{Kec}$ and $\mathrm{Ku}$, and value of them is $1,1,0.1$ respectively. Membership degree of the error, the error change rate and the output, respectively showed in Table 1, Table 2 and Table 3.

Table 1. Membership Degree of the Error

\begin{tabular}{|c|c|c|c|c|c|c|c|}
\hline $\begin{array}{ll}\text { variable } & E \\
\end{array}$ & $\mathbf{0}$ & 1 & 2 & 3 & 4 & 5 & 6 \\
\hline VB & & & & & 0.4 & 0.7 & 1 \\
\hline B & & & & 0.4 & 0.7 & 1 & 0.7 \\
\hline MB & & & 0.4 & 0.7 & 1 & 0.7 & \\
\hline $\mathbf{M}$ & & 0.4 & 0.7 & 1 & 0.7 & & \\
\hline MS & 0.4 & 0.7 & 1 & 0.7 & & & \\
\hline $\mathbf{S}$ & 0.7 & 1 & 0.7 & & & & \\
\hline VS & 1 & 0.7 & & & & & \\
\hline
\end{tabular}

Table 2. Membership Degree of the Error Change Rate

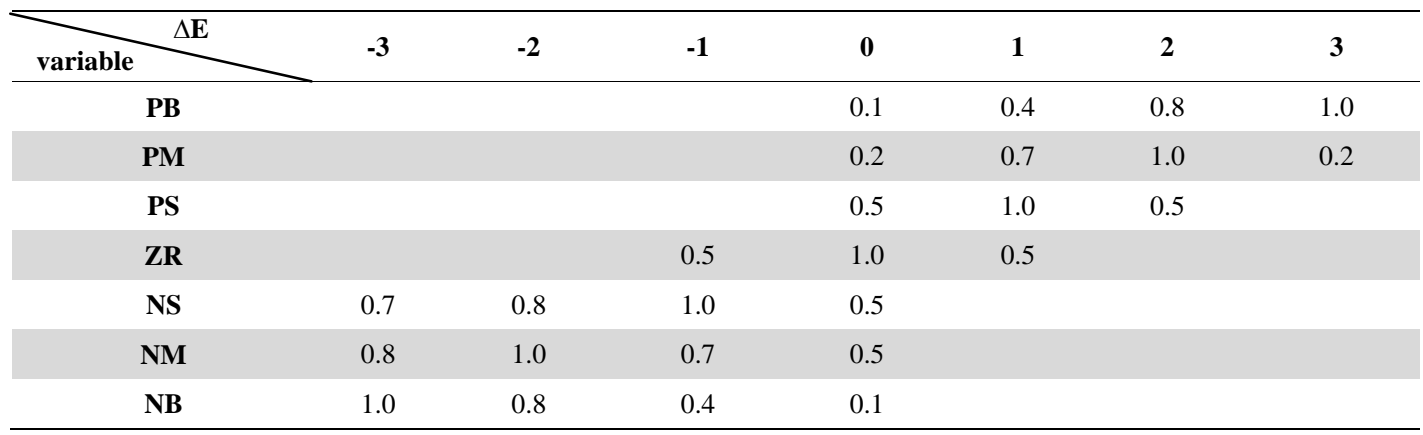

Table 3. Membership Degree of the Output

\begin{tabular}{cccccccc}
\hline variable & $\mathbf{U}$ & $\mathbf{0}$ & $\mathbf{2}$ & $\mathbf{3}$ & $\mathbf{4}$ & $\mathbf{5}$ & $\mathbf{6}$ \\
\hline VB & & & & 0.4 & 0.7 & 1 \\
$\mathbf{B}$ & & & 0.4 & 0.7 & 1 & 0.7 \\
MB & & & 0.4 & 0.7 & 1 & 0.7 & \\
M & 0.4 & 0.7 & 1 & 0.7 & & & \\
MS & 0.7 & 1 & 0.7 & & & & \\
S & 1 & 0.7 & & & & \\
VS & & & & & & & \\
\hline
\end{tabular}




\subsection{Generation of fuzzy rules}

Fuzzy rules are generated by repeated experiments according to work experiences, shown in Table 4 .

Table 4. Table of Fuzzy Rule

\begin{tabular}{cccccccccc}
\hline \hline $\mathbf{E}$ & $\mathbf{E}$ & VS & S & MS & M & MB & B & VB \\
\hline & PB & VS & VS & VS & S & MS & M & MB \\
PM & VS & VS & S & MS & M & MB & B \\
PS & VS & S & MS & M & MB & B & VB \\
ZR & VS & S & MS & M & MB & B & VB \\
NS & VS & MS & M & M & MB & B & VB \\
NM & VS & M & M & MB & B & VB & VB \\
NB & VS & MB & B & B & VB & VB & VB \\
\hline
\end{tabular}

\subsection{Generation of fuzzy control query table}

Fuzzy control query table is generated by using Mamdani algorithm and defuzzification is the largest membership degree method, shown in Table 5.

Table 5. Fuzzy Control Query Table

\begin{tabular}{rlllllll}
\hline$\Delta \mathbf{E}$ & $\mathbf{0}$ & $\mathbf{1}$ & $\mathbf{3}$ & $\mathbf{4}$ & $\mathbf{5}$ & $\mathbf{6}$ \\
\hline $\mathbf{- 3}$ & 0 & 1 & 2 & 2 & 3 & 4 & 4 \\
$\mathbf{- 2}$ & 0 & 1 & 2 & 2 & 3 & 4 & 4 \\
$\mathbf{- 1}$ & 0 & 2 & 3 & 4 & 4 & 5 & 5 \\
$\mathbf{0}$ & 0 & 2 & 3 & 4 & 5 & 5 & 6 \\
$\mathbf{1}$ & 0 & 3 & 4 & 5 & 5 & 5 & 6 \\
$\mathbf{2}$ & 0 & 4 & 5 & 5 & 5 & 6 & 6 \\
\hline $\mathbf{3}$ & 0 & 4 & 5 & 5 & 6 & 6 & 6
\end{tabular}

\section{Temperature Control}

The fuzzy controller looks up table 5 according to the error and its change rate, then output of fuzzy controller is generated, which is multiplied by the scaling factor. Because output of fuzzy controller is the duty cycle of pulse sequence, the timer produces a corresponding duty-cycle pulse sequence. The temperature of the oven is changed by controlling the time of oven power supply, which is completed by a solid-state relay.

Response curve of the oven temperature is shown in Fig. 4. 


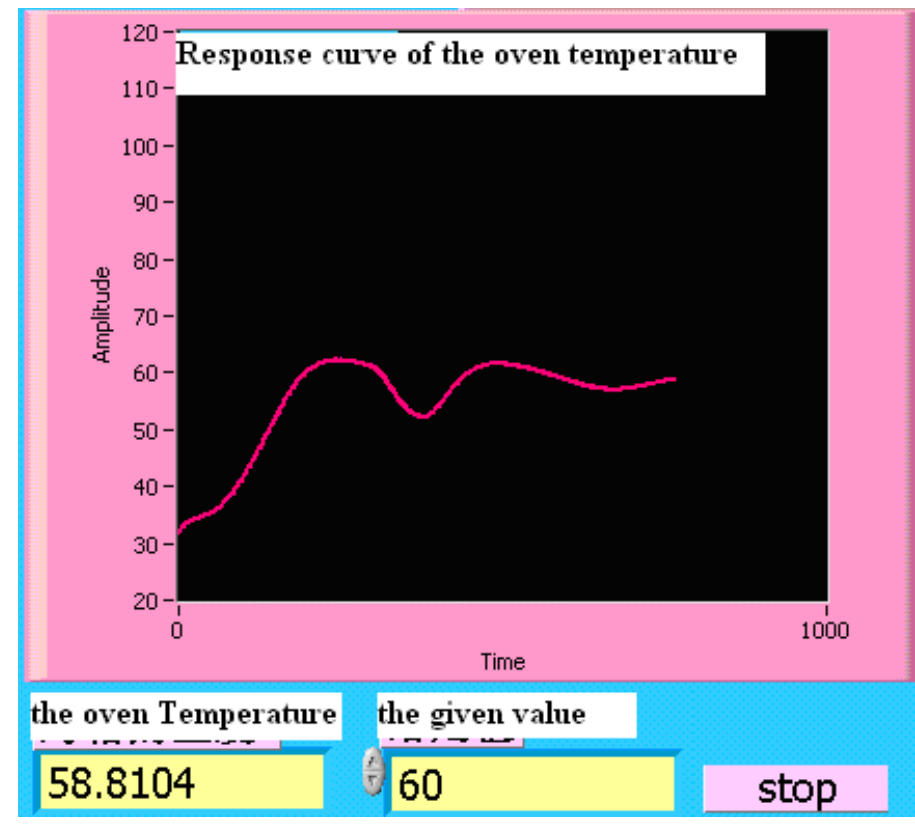

Fig. 4. Response curve of the oven temperature

\section{Conclusion}

In this paper, fuzzy temperature control system is designed based on LabVIEW. Using the graphical programming approach and fuzzy logic, this system has a good robustness and dynamic performance and obtain better control effect in practice. Design process of the fuzzy control is simple and convenient, which provides a new way for designing fuzzy control system.

\section{Acknowledgements}

The work is supported by research project of Beijing Union University and College of Automation of Beijing Union University. (No.zk200920x and 1102541602).

\section{References}

[1] Yang Leping, Li Haitao, Yang Lei. LabVIEW Program Design and Application [M]. Beijing: Electronic Industry Press, 2005.(in Chinese)

[2] Hou Ping, Ye Xin Qi. LabVIEW7.1 programming and virtual instrument design [M]. Beijing: QingChinese University Press, 2005.(in Chinese)

[3] Liu Junhua, et al. Virtual Instruments Labview graphical programming language tutorial [M]. Xi'an: Xidian University Press, 2001.(in Chinese)

[4] Li-yong. Control, neural control and intelligent control theory [M]. Harbin: Harbin Institute of Technology Press, 1996.(in Chinese) 